\title{
Multimethodological approach to the study of carbide particle dimensions in high-strength steel
}

\author{
M. L. Fedoseev ${ }^{1, \dagger}$, S. N. Petrov ${ }^{1}$, A. Kh. Islamov², N. F. Drozdova ${ }^{1}$, T. A. Lychagina ${ }^{2}$, \\ D. I. Nikolayev ${ }^{2}$ \\ ${ }^{\dagger}$ fedoseevml@hotmail.com \\ ${ }^{1}$ NRC “Kurchatov institute” - CRISM “Prometey”, 49 Spalernaya str., St. Petersburg, 190015, Russia \\ ${ }^{2}$ Joint Institute for Nuclear Research, 6 Joliot-Curie str., Dubna, 141980, Russia
}

\begin{abstract}
An important task in developing new materials is the study of their structure (substructure). The present study uses a number of complementary methods to determine the size, morphology, phase composition and volume fraction of dispersed precipitates in high-strength medium-carbon steel tempered in the temperature range of up to $600^{\circ} \mathrm{C}$. The methods of small-angle neutron scattering, X-ray and neutron diffraction were used. Such an advantage of neutrons as the ability to use large samples, which significantly increases the maximum permissible grain size to obtain statistically reliable results and takes into account texture data, explains the use of neutron sources along with the X-ray ones. The disadvantages include a lower resolution and more complex equipment. The combined use of different techniques made it possible to determine the kinetics of structural changes in the dispersed particles during tempering of steel. The increase in tempering temperature up to $300^{\circ} \mathrm{C}$ results in a decrease in the amount of retained austenite. At the tempering temperature of $300^{\circ} \mathrm{C}$, the growth of $\mathrm{Fe}_{3} \mathrm{C}$ particles instead of retained austenite starts and continues up to $600^{\circ} \mathrm{C}$ up to which the studies were done. The phases of retained austenite and cementite were identified by means of neutron diffraction, while changes in sizes were determined by means of small-angle neutron scattering. The results obtained do not contradict the previous transmission electron microscopy data. A comprehensive study by means of diffraction and scattering methods of X-rays and neutrons allows one to obtain exhaustive information about disperse particles in steels.
\end{abstract}

Keywords: small-angle neutron scattering, X-Ray and neutron diffraction, carbides, high-strength steel.

УДК: 539.26:539.27

\section{Комплексный подход к количественному описанию карбидов в высокопрочной стали}

\author{
Федосеев М. Л. ${ }^{1, \dagger}$, Петров С.Н. ${ }^{1}$, Исламов А. Х², Дроздова Н. $\Phi^{1}$, Лычагина Т. А², \\ Николаев Д. И. ${ }^{2}$ \\ ${ }^{1}$ НИЦ «Курчатовский институт» - ЦНИИ КМ «Прометей», ул. Шпалерная 49, Санкт-Петербург, 190015, Россия \\ ${ }^{2}$ Объединенный институт ядерных исследований (ОИЯИ), ул. Жолио-Кюри 6, Дубна, 141980, Россия
}

Важной задачей при создании новых материалов является изучение структуры (субструктуры). В данном исследовании использован ряд взаимодополняющих методов, позволяющих определить размер, морфологию, фазовый состав и объемную долю дисперсных выделений в высокопрочной среднеуглеродистой стали, подвергнутой различным отпускам в диапазоне температур до $600^{\circ} \mathrm{C}$. Применены методы: малоуглового нейтронного рассеяния, рентгеновской и нейтронной дифракции. Использование наряду с рентгеновскими источниками нейтронных обусловлено рядом присущих им особенностей. К преимуществам нейтронов можно отнести возможность использовать большие образцы, при этом значительно увеличивается предельно допустимый размер зерна для получения статистически достоверных результатов, также значительно упрощается учет влияния текстуры. К недостаткам можно отнести меньшее разрешение и более сложное оборудование. Совместное использование взаимодополняющих методов позволило установить кинетику структурных изменений дисперсных выделений при отпуске стали. Так, рост температуры отпуска до $300^{\circ} \mathrm{C}$ приводит к уменьшению количества остаточного аустенита. При температуре отпуска $300^{\circ} \mathrm{C}$ начинается и продолжается вплоть до $600^{\circ} \mathrm{C}$ (далее не исследовалось) рост частиц $\mathrm{Fe}_{3} \mathrm{C}$ вместо остаточного аустенита. При этом фазы остаточного аустенита и цементита установлены при помощи нейтронной дифракции. А измене- 
ния размеров дисперсных частиц фаз установлены при помощи малоуглового нейтронного рассеяния. Результаты не противоречат ранее полученным данным локального метода просвечивающей электронной микроскопии. Таким образом, комплексное исследование при помощи методов дифракции и рассеяния рентгеновского излучения и нейтронов позволяет получать достаточно полную информацию о дисперсных выделениях в сталях.

Ключевые слова: малоугловое нейтронное рассеяние, рентгеновская и нейтронная дифракция, карбиды, высокопрочная сталь.

\section{1. Введение}

При разработке новых сталей весьма актуален вопрос об определении размера, морфологии, фазового состава и объемной доли дисперсных выделений наномасштабного диапазона размеров, так как они напрямую влияют на свойства получаемой стали. Ни один из существующих сегодня методов не обеспечивает определения всех заявленных выше характеристик, поскольку для определения размеров, морфологии и фазового состава необходима высочайшая локальность и пространственное разрешение, тогда как для корректного определения объемной доли необходимо исследование значительного объема материала.

В связи с этим представляется целесообразным комплексное использование ряда методов, одни из которых обладают достаточной локальностью и пространственным разрешением, а другие обеспечивают получение интегральных характеристик. В частности, использование методов дифракции и рассеяния рентгеновского и нейтронного излучений в сочетании с таким высоколокальным методом как просвечивающая электронная микроскопия (ПЭМ). Дифракционные методы позволяют получить информацию об интегральном фазовом составе образца с пределом обнаружения до долей процента. Малоугловое рассеяние несет данные о размере и форме дисперсных выделений до 100 нм, при этом фазовый состав остается неизвестен. ПЭМ, в свою очередь, позволяет определить размеры и морфологию, а также идентифицировать фазовый состав отдельных частиц. Комплексный анализ информации, получаемой различными методами, позволяет достоверно аттестовать состояние дисперсных выделений на различных этапах технологического передела.

Привлечение к проведению исследований как рентгеновского, так и нейтронного излучений также обеспечивает получение дополнительной информации. Нейтроны взаимодействуют с ядрами атомов в отличии от рентгеновского излучения, взаимодействующего с электронными оболочками [1]. Это приводит к тому, что амплитуда взаимодействия нейтронов с материалом исследования табулирована и меняется случайным образом от атома к атому, тогда как амплитуда рентгеновского взаимодействия увеличивается пропорционально числу электронов мишени. Слабая абсорбция нейтронов в исследуемом материале обеспечивает значительно больший объем исследования, чем при использовании рентгеновского излучения.

В данной работе на примере высокопрочной среднеуглеродистой стали проведено исследование состояния дисперсных выделений при различных температурах отпуска с использованием комплекса методов, обладающих разной локальностью и репрезентативностью.

\section{2. Материалы и методы исследования}

Химический состав и механические свойства исследуемой высокопрочной среднеуглеродистой стали представлены в Табл. 1-2 [2-5]:

Табл. 1. Химический состав исследуемой стали (для Са и В по расчету).

Table 1. Chemical composition of the steel (for Са и В - by calculation).

\begin{tabular}{|c|c|c|c|c|c|c|c|c|c|}
\hline \multirow{2}{*}{\multicolumn{2}{|c|}{$\begin{array}{l}\text { Марка стали } \\
\text { Steel grade }\end{array}$}} & \multicolumn{8}{|c|}{$\begin{array}{c}\text { Массовая доля элементов, \% } \\
\text { Mass fraction of elements, \% }\end{array}$} \\
\hline & & \multirow{2}{*}{$\frac{\mathrm{C}}{0.4}$} & \multirow{2}{*}{$\frac{\mathrm{Si}}{0.2}$} & \multirow{2}{*}{$\frac{\mathrm{Mn}}{1.1}$} & \multirow{2}{*}{\multicolumn{2}{|c|}{$\begin{array}{l}\mathrm{Cr} \\
0.9\end{array}$}} & \multirow{2}{*}{$\frac{\mathrm{Ni}}{0.65}$} & \multirow{2}{*}{$\begin{array}{l}\mathrm{Cu} \\
0.5\end{array}$} & \multirow{2}{*}{$\begin{array}{l}\text { Mo } \\
0.35\end{array}$} \\
\hline \multirow{2}{*}{ Б1700 } & $\min$ & & & & & & & & \\
\hline & $\max$ & 0.45 & 0.45 & 1.3 & & & 0.8 & 0.8 & 0.45 \\
\hline \multirow{2}{*}{\multicolumn{2}{|c|}{$\begin{array}{c}\text { Марка стали } \\
\text { Steel grade }\end{array}$}} & \multicolumn{8}{|c|}{$\begin{array}{c}\text { Массовая доля элементов, \% } \\
\text { Mass fraction of elements, \% }\end{array}$} \\
\hline & & V & $\mathrm{Nb}$ & A & & $\mathrm{Ti}$ & & $\mathrm{Ca}$ & B \\
\hline \multirow{2}{*}{ Б1700 } & $\min$ & 0.04 & 0.01 & 0.0 & & 0.0 & & 0.001 & 0.001 \\
\hline & $\max$ & 0.06 & 0.04 & 0.0 & & 0.0 & & 0.02 & 0.005 \\
\hline
\end{tabular}

Табл. 2. Требования (минимальные) к механическим свойствам исследуемой стали.

Table 2. Requirements for the mechanical properties of the steel.

\begin{tabular}{|c|c|c|c|}
\hline$\sigma_{0.2}, \mathrm{MPa}$ & $\sigma_{\mathrm{z}}, \mathrm{MPa}$ & $\delta_{5}, \%$ & $\mathrm{KCU}^{20}, \mathrm{~J} / \mathrm{cm}^{2}$ \\
\hline 1700 & 1850 & 7 & 20 \\
\hline
\end{tabular}

Закалка и определение температур фазовых превращений проведены с использованием дилатометра Dil-805 BAHR Thermoanalyse. Использован следующий режим: закалка с температуры $900^{\circ} \mathrm{C}$, нагрев до $700^{\circ} \mathrm{C}$ со скоростью $1 \%$ мин. Малая скорость нагрева выбрана для получения наиболее корректных температур фазовых превращений. Поскольку эффекты дилатации при превращении дисперсных фаз невелики, для выявления температур фазовых превращений использованы дифференциальные дилатометрические кривые, полученные аналогично [6-8], а также кривые, отображающие отклонение от линии дилатации, аппроксимированной прямой. Данная кривая получается значительно проще, чем дифференциальная кривая, кроме того, для нее не требуется проведения усреднения, так как она не очень чувствительна к разбросу исходных данных. Вследствие этого ее удобно использовать для первоначальной оценки критических температур.

Исследования фазового состава образцов после различных температур отпуска были проведены на много- 
функциональном рентгеновском дифрактометре Rigaku Ultima IV с использованием программного комплекса PDXL (X-ray Powder Diffraction Software) с базой порошковых дифракционных стандартов PDF-2.

Для исследований при помощи нейтронов использованы установки на импульсном реакторе ИБР-2 в ОИЯИ [9]. Для выявления мелкодисперсных карбидных составляющих стали был использован нейтронный текстурный дифрактометр СКАТ. На дифрактометре реализована времяпролетная (time-of-flight) методика измерений [10 - 12]. Временная развертка такого сигнала является времяпролетным дифракционным спектром. Полное время пролета нейтрона связано с длиной волны $\lambda$ следующим выражением: $t=L \lambda m / h$, где $m-$ масса нейтрона, $h-$ постоянная Планка, $L-$ полная пролетная база. Поэтому в детекторе, расположенном под углом $2 \theta$ по отношению к падающему пучку, в силу условия Брэгга $\lambda=d_{h k l} \sin (\theta)$ во временных каналах, соответствующих временам $t_{h k l}=L d_{h k l} \sin (\theta) m / h$, будут наблюдаться дифракционные максимумы. Так как детекторные модули расположены на кольце под углами от $0^{\circ}$ до $180^{\circ}$, одного вращения вокруг горизонтальной оси гониометра достаточно для измерения полных полюсных фигур. При измерении образцов на текстурном дифрактометре СКАТ проводилось полное ориентационное усреднение. Использование суммарных спектров позволило уменьшить ошибки, связанные с неизбежным присутствием текстуры в образцах.

Данные о размерах дисперсных выделений были получены при помощи малоуглового нейтронного рассеяния (МУНР). Эксперименты по малоугловому нейтронному рассеянию образцов стали проведены с использованием времяпролетного спектрометра ЮМО, [13-14]. Исследования проведены на расстоянии образец - детектор 5.28 и 13.04 м, обеспечивающем диапазон получаемых значений вектора рассеяния $Q$ от 0.007 до $0.5 \AA^{-1}$. Облучаемый диаметр образца составил 14 мм. Интенсивности рассеяния нейтронов на полученных спектрах приведены к абсолютным единицам $\mathrm{cm}^{-1}$ путем корректировки интенсивности рассеяния на поглощение и толщину образца, фоновое рассеяние на подложке и на контрольный образец из ванадия при помощи ПO SAS [15-16].

В исследуемых образцах изменения вектора рассеяния аппроксимируются законом Порода, отвечающим рассеянию от объектов с гладкой поверхностью, объёмом $V$ и площадью $S$, при этом интенсивность рассеяния:

$$
I(Q)=A \cdot Q^{-4}=2 \pi \cdot \Delta \rho^{2} \cdot S / V \cdot Q^{-4} .
$$

где $\Delta \rho=\rho_{\mathrm{p}}-\rho_{\mathrm{s}}-$ контраст, $\rho_{\mathrm{p}}-$ плотность рассеяния частицы, $\rho_{\mathrm{s}}-$ плотность рассеяния матрицы, в которой находятся частицы.

Получая значение коэффициента $A$ из эксперимента и предполагая в первом приближении объект сферическим можно получить значение радиуса $R$ частицы:

$$
R=2 \pi \cdot \Delta \rho^{2} \cdot 3 / A \text {. }
$$

Рассматривая объект как прямоугольный параллелепипед со сторонами $a, b$ и $c$, когда размеры $b$ и $c$ намного больше $a$ (рейка):

$$
a=2 \pi \cdot \Delta \rho^{2} \cdot 2 / A \text {. }
$$

Следует заметить, что метод малоуглового нейтронного рассеяния (МУРН) требует относительно малого времени эксперимента (1 образец порядка 30 мин), в связи с чем исследования проведены в достаточно широком интервале температур отпуска от 150 до $600^{\circ} \mathrm{C}$ с шагом по температуре отпуска не более $50^{\circ} \mathrm{C}$, что позволяет, наряду с дилатометрией, рассмотреть весь исследуемый интервал температур отпуска.

\section{3. Результаты исследований}

\section{1. Дилатометрия}

Для более подробного изучения дилатометрической кривой построены дифференциальные дилатограммы [6]. Выявлены три критические точки температур на дилатометрической кривой: $\sim 130,280,380^{\circ} \mathrm{C}$, соответствующие превращениям фаз остаточного аустенита и цементита при отпуске стали [17-19], [7-8] (Рис. 1).

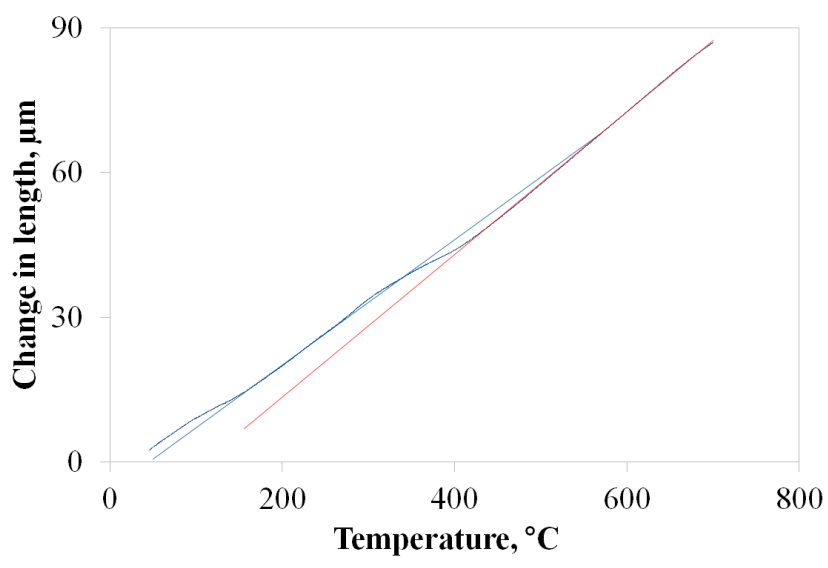

(а) дилатометрическая кривая

(a) dilatometric curve

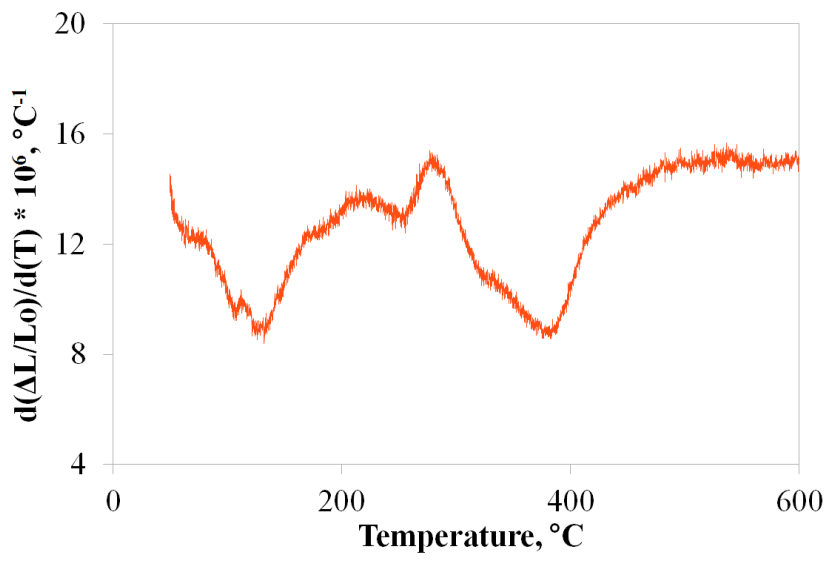

(b) дифференциальная дилатометрическая кривая

(b) differential dilatometric curve

Рис. 1. Изменение длины образца после закалки, скорость нагрева $1^{\circ} \mathrm{C} / \mathrm{min}$.

Fig. 1. Change in length of specimens after quenching with a heating rate $1^{\circ} \mathrm{C} / \mathrm{min}$. 


\section{2. Малоугловое рассеяние нейтронов}

На Рис. 2 представлены кривые малоуглового нейтронного рассеяния стали после закалки (обозначен $20^{\circ} \mathrm{C}$ ) и при температурах отпуска в диапазоне от $150^{\circ} \mathrm{C}$ до $600^{\circ} \mathrm{C}$.

В области больших значений векторов рассеяния $0.2 \AA^{-1}<Q<0.5 \AA^{-1}$, соответствующих шкале размеров $d \sim 2 \pi / Q$ от 1 нм до 3 нм, практически нет изменений на кривых рассеяния, также как и для малых значений $Q<0.009 \AA^{-1}$, соответствующих размеру 70 нм. Это может означать, что основная структура стали (зёренная структура) существенно не изменяется в данном диапазоне температур.

Основные изменения на кривых рассеяния происходят в области векторов рассеяния $0.009 \AA^{-1}<Q<0.12 \AA^{-1}$, которая хорошо аппроксимируется законом рассеяния Порода, описывающим рассеяние от объектов с гладкой поверхностью.

Падение интенсивности малоуглового нейтронного рассеяния начинается при температуре отпуска $150^{\circ} \mathrm{C}$ и заканчивается при $250^{\circ} \mathrm{C}$, далее вплоть до $390^{\circ} \mathrm{C}$ кривые рассеяния остаются практически неизменными, а после $390^{\circ} \mathrm{C}$ стремятся обратно к кривой закалки и достигают ее уровня при температурах отпуска $500-600^{\circ} \mathrm{C}$.

На основе дилатометрических и кривых малоуглового рассеяния выбраны три критические температуры отпуска для дальнейшего исследования методами нейтронной и рентгеновской дифракции, а именно: $150^{\circ} \mathrm{C}-$ начало падения интенсивности малоуглового рассеяния, $300^{\circ} \mathrm{C}-$ стагнация и $550^{\circ} \mathrm{C}-$ конец роста интенсивности.
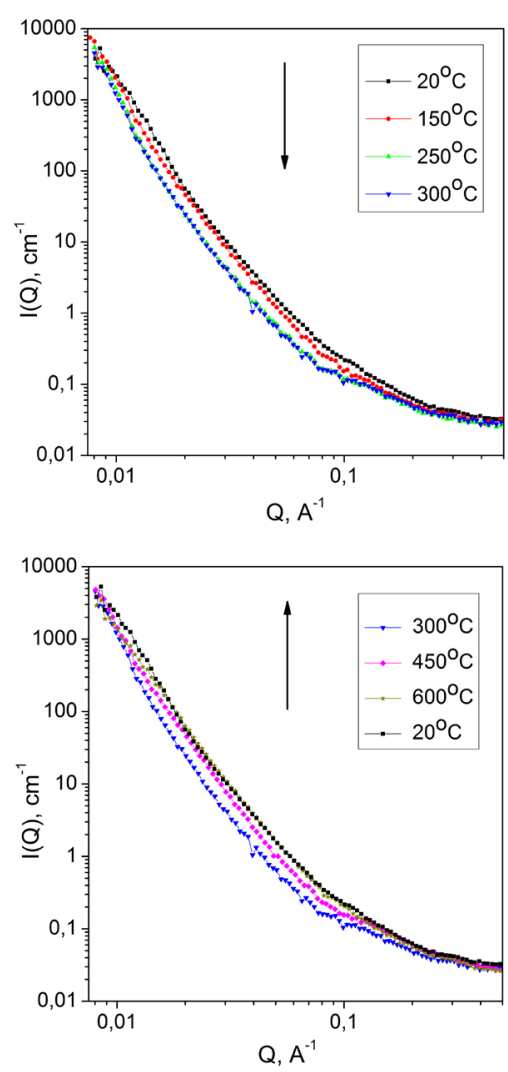

Рис. 2. Кривые малоуглового рассеяния нейтронов на образцах. Fig. 2. SANS curves of specimens.

\section{3. Рентгеноструктурный анализ (РСА)}

По результатам РСА фазовый состав образцов не изме-

Табл. 3. Параметры и микроискажения кристаллической решетки образцов.

Table 3. Lattice parameters and crystalline strain of the samples.

\begin{tabular}{|c|c|c|}
\hline $\begin{array}{c}\text { Температура отпуска, }{ }^{\circ} \mathrm{C} \\
\text { Temperature of tempering }\end{array}$ & $\begin{array}{c}\text { Микроискажения, \% } \\
\text { Crystalline strain }\end{array}$ & $a, \AA$ \\
\hline 150 & 0.55 & 2.871 \\
\hline 300 & 0.35 & 2.869 \\
\hline 500 & 0.19 & 2.869 \\
\hline
\end{tabular}

нился при изменении температуры отпуска. В дифракционном спектре присутствует лишь одна фаза $-\alpha$-Fe, параметр и микроискажения кристаллической решетки которой уменьшаются при увеличении температуры отпуска, см. Табл. 3.

Уменьшение микроискажений кристаллической решетки $\alpha$-Fe при повышении температуры отпуска связано с переходом в более равновесное состояние. Некоторое уменьшение параметра кристаллической решетки связано с обеднением матрицы по углероду, вызванным выделением карбидов (туда уходит избыточный углерод из матрицы, уменьшая при этом уровень микроискажений).

\section{4. Дифракция нейтронов}

Исследованы образцы стали, отвечающие различным температурам отпуска, выбранным в соответствии с описанными выше исследованиями. В фазовом составе образцов кроме основной фазы $\alpha$-Fе также обнаружены фазы $\gamma$-Fe и $\mathrm{Fe}_{3} \mathrm{C}$. При этом количество остаточного аустенита в зависимости от повышения температуры отпуска от $150^{\circ} \mathrm{C}$ к $300^{\circ} \mathrm{C}$ падает с $3 \%$ до $0.2 \%$ [20] (Рис. 3a), в то время как карбид $\mathrm{Fe}_{3} \mathrm{C}$, зафиксированный при температуре $400^{\circ} \mathrm{C}$ на грани выявляемости, при повышении температуры до $550^{\circ} \mathrm{C}$ уже различим и имеет ряд независимых пиков (см. Рис. 3b).

\section{4. Обсуждение результатов}

Плотности рассеяния найденных нейтронной дифракцией фаз остаточного аустенита (гранецентрированная кубическая (ГЦК) кристаллическая решетка) $\sim 8.8 \times 10^{10} \mathrm{~cm}^{-2}$ и цементита $\sim 8.7 \times 10^{10} \mathrm{~cm}^{-2}$ дают достаточно близкие значения контраста с матрицей из мартенсита (объемноцентрированная кубическая (ОЦК) кристаллическая решетка) $8 \times 10^{10} \mathrm{~cm}^{-2}$. Следует заметить, что плотности рассеяния чистого железа и железа в случае полной растворимости $0.42 \%$ весовых процента углерода практически не отличаются.

Из Рис. 2 видно, что падение интенсивности малоуглового нейтронного рассеяния начинается при темпера- 


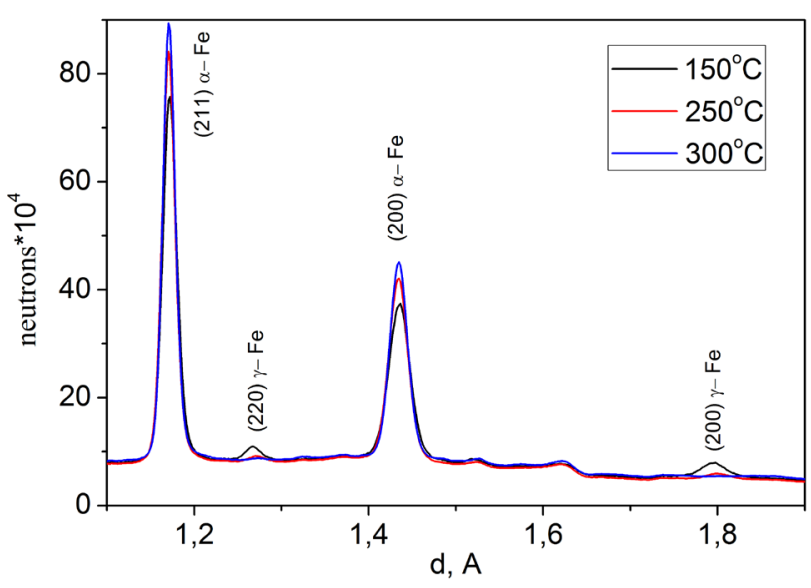

a

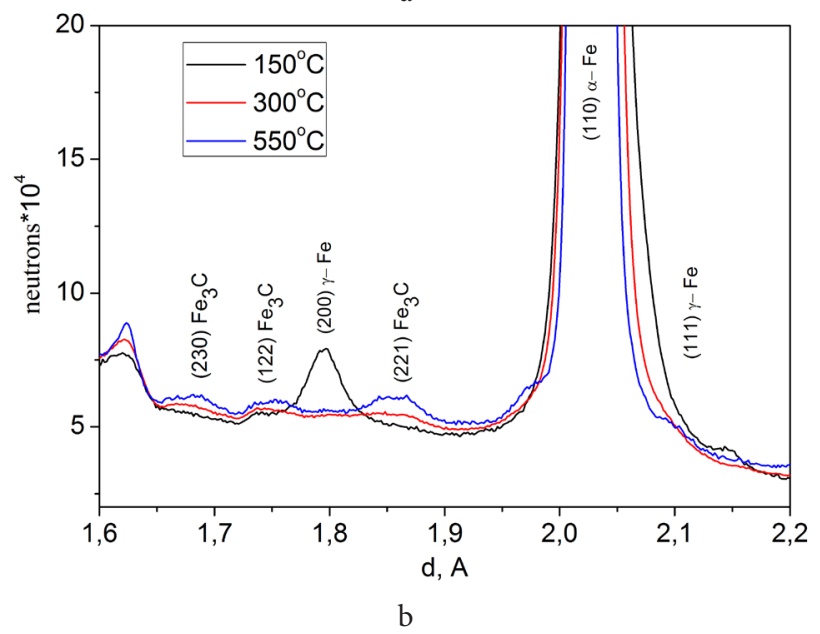

Рис. 3 Нейтронная дифракции образцов после различных температур отпуска: до $300^{\circ} \mathrm{C}$ (a), до $550^{\circ} \mathrm{C}(\mathrm{b})$.

Fig. 3 Neutron diffraction of the samples after different tempering temperatures: to $300^{\circ} \mathrm{C}$ (a), to $550^{\circ} \mathrm{C}(\mathrm{b})$.

туре отпуска образца $150^{\circ} \mathrm{C}$ и заканчивается при $250^{\circ} \mathrm{C}$. Опираясь на данные нейтронной дифракции можно предположить, что при $150{ }^{\circ} \mathrm{C}$ начинается распад остаточного аустенита с образованием мартенсита отпуска.

В диапазоне температур отпусков $250^{\circ} \mathrm{C}-390^{\circ} \mathrm{C}$ кривые рассеяния остаются практически неизменными, что свидетельствует об окончании превращения остаточного аустенита, при этом по границам реек мартенсита в местах существования остаточного аустенита начинает формироваться $\mathrm{Fe}_{3} \mathrm{C}$.

После $390^{\circ} \mathrm{C}$ кривые рассеяния стремятся обратно к кривой закалки. Кривые рассеяния при температурах отпуска $500-600^{\circ} \mathrm{C}$ сравниваются с кривой рассеяния образца после закалки в связи с тем, что плотность рассеяния (контраст) $\gamma$-Fе практически равна $\mathrm{Fe}_{3} \mathrm{C}$. Растет содержание $\mathrm{Fe}_{3} \mathrm{C}$ по границам реек мартенсита, что также соответствует данным нейтронной дифракции. Дальнейший рост температуры отпуска не оправдан в связи с тем, что повышение температуры приводит к выделению $\mathrm{Fe}_{3} \mathrm{C}$ по границам зерен, приводя к отпускной хрупкости.

На Рис. S1 (дополнительный материал) приведены дополнительные вклады в рассеяние от основной кривой при $300^{\circ} \mathrm{C}$, полученные путем вычитания. Эти вклады удовлетворяют закону рассеяния Порода. Из уравнений 2-3 можно определить характерные размеры прослоек аустенита и частиц цементита. В случае приближения формы частицы шаром линейный размер соответствует 35 нм. В случае рейки, где два размера намного больше, чем первый размер $\sim 12$ нм. Т.е. размер рассеивающих частиц (аустенита или цементита) находится в диапазоне от 12 до 35 нм в зависимости от выбранной формы аппроксимирующих частиц.

При этом согласно [4] по данным ПЭМ в исходном состоянии преобладает реечный мартенсит с шириной рейки $\sim 100$ нм, а остаточный аустенит расположен в качестве прослоек между реек мартенсита шириной около 50 нм.

Таким образом, при падении интенсивности малоуглового нейтронного рассеяния объектом рассеяния является остаточный аустенит, а матрицей - мартенсит, а при повышении интенсивности рассеяния до прежних значений объектом рассеяния служит уже $\mathrm{Fe}_{3} \mathrm{C}$.

\section{5. Заключение}

В результате проведенных исследований установлена кинетика структурных изменений среднеуглеродистой стали в зависимости от температуры отпуска. Исходная структура после закалки представляет собой преимущественно реечный мартенсит с вкраплениями остаточного аустенита между рейками. Рост температуры отпуска приводит к уменьшению количества остаточного аустенита (изменение фазового состава и падение интенсивности рассеяния). Падение интенсивности нейтронного рассеяния стабилизируется в области температур отпуска $\sim 300^{\circ} \mathrm{C}$ в связи с практически полным распадом остаточного аустенита. Дальнейший рост температуры отпуска вплоть до $600^{\circ} \mathrm{C}$ приводит к росту интенсивности рассеяния, и кривая отпуска при $600^{\circ} \mathrm{C}$ совпадает с кривой после закалки. Данный эффект можно связать с тем, что по мере увеличения температуры отпуска остаточный аустенит заменяется фазой $\mathrm{Fe}_{3} \mathrm{C}$ с примерно такой же плотностью рассеяния.

Полученные результаты наглядно иллюстрируют преимущества комплексного применения взаимодополняющих методов дифракции и рассеяния рентгеновского излучения, электронов и нейтронов, что позволяет получать достаточно полную информацию о дисперсных выделениях в сталях и выбирать температуру отпуска (в общем случае корректировать термообработку), исходя из предъявляемых требований.

Благодарности/Acknowledgements. Экспериментальные исследования выполнены на оборудовании Центра коллективного пользования научным оборудованием «Состав, структура и свойства конструкиионных и функциональных материалов» НИЦ «Курчатовский институт» - ЦНИИ КМ «Прометей» при финансовой поддержке Минобрнауки России в рамках соглашения №14.595.21.0004, уникальный идентификатор RFMEFI59517X0004./The research was carried out in the Center for Common Facilities on the unique equipment "Composition, structure and properties of structural and 
functional materials" of NRC "Kurchatov institute" - CRISM "Prometey", supported by the Russian Ministry of Education and Science (project № 14.595.21.0004, unique identifier RFMEFI59517X0004).

Дополнительныйматериал/SupplementaryMaterial. Электронная версия статьи содержит дополнительный материал (рисунок), доступньй безвозмездно на сайте журнала (www.lettersonmaterials.com)./The online version of this paper contains supplementary material (figure) available free of charge at the journal's Web site (www.lettersonmaterials.com).

\section{Литература/References}

1. F. Cousin. Small angle neutron scattering. EPJ Web of Conferences 104, 01004 (2015). DOI: $10.1051 /$ epjconf/201510401004

2. V.V. Ryabov, E.I. Khlusova, S.A. Golosienko, G. D. Motovilina. Metallurg. 6, 59 (2015). (in Russian) [В.В. Рябов, Е.И. Хлусова, С.А. Голосиенко, Г. Д. Мотовилина. Металлург. 6, 59 (2015).]

3. V. V. Ryabov, G. D. Motovilina, E. I. Khlusova, S. A. Sidorov. Metallurg. 8, 72 (2016). (in Russian) [В.В. Рябов, Г.Д. Мотовилина, Е.И. Хлусова, С.А. Сидоров. Металлург. 8, 72 (2016).]

4. V. V. Ryabov. Razrabotka iznosostoykoy stali s predelom tekuchesti 1200-1700 MPa dlya detaley rabochih organov pochvoobrabatyvayuschih mashin: Dissertacija na soiskanie stepeni kandidata tehnicheskih nauk. St. Petersburg. (2016) 235 p. (in Russian) [В. В. Рябов. Разработка износостойкой стали с пределом текучести 1200 - 1700 МПа для деталей рабочих органов почвообрабатывающих машин: дисс. канд. техн. наук. Санкт-Петербург. (2016) 235 с.]

5. A.Yu. Izmailov, S.A. Sidorov, V.K. Khoroshenkov, E.I. Khlusova, V.V. Ryabov. Metal Science and Heat Treatment. 4(742), 13 (2017). (in Russian) [А. Ю. Измайлов, С.А. Сидоров, В.К. Хорошенков, Е.И. Хлусова, В.В. Рябов. Металловедение и термическая обработка металлов. 4(742), 13 (2017).]

6. J. Pacyna Journal of Achievements in Materials and Manufacturing Engineering. 46, 7 (2011).

7. M.S. Mikhailov, V.V. Ryabov. Material Science and Technology News. 19(1), 3 (2016). (in Russian) [М. С. Михайлов, В.В. Рябов. Новости материаловедения. Наука и техника. 19(1), 3 (2016).]
8. V.V. Ryabov, T.V. Knyazyuk, M.S. Mikhailov, G.D. Motovilina, E.I. Khlusova. Voprosy Materialovedeniya (Problems of Materials Science). 86(2), 7 (2016). (in Russian) [В. В. Рябов, Т. В. Князюк, М.С. Михайлов, Г.Д. Мотовилина, Е.И. Хлусова. Вопросы материаловедения. 86(2), 7 (2016).]

9. V.N. Shvetsov. Quantum Beam Science. 6(1), 1 (2017). DOI: $10.3390 /$ qubs 1010006

10. R. Keppler, K. Ullemeyer, J.H. Behrmann, M. Stipp. Journal of Applied Crystallography. 47, 1520 (2014). DOI: 10.1107/S1600576714015830

11. D.I. Nikolayev, T.A. Lychagina, A. V. Nikishin, V.V. Yudin. Solid State Phenomena. 105, 77 (2005). DOI: 10.4028/www.scientific.net/SSP.105.77

12. T. Lychagina, D. Nikolayev, A. Sanin, J. Tatarko, K. Ullemeyer. 17th International Conference on Textures of Materials (ICOTOM 17). 82(012107), 1 (2015). DOI: $10.1088 / 1757-899 X / 82 / 1 / 012107$

13. Y.M. Ostanevich. Macromol. Chem. Macromol. Symp. 15, 91 (1988). DOI: 10.1002/masy.19880150107

14. A. I. Kuklin, A. K. Islamov, V. I. Gordeliy. Neutron News. 3(16), 16 (2005). DOI: 10.1080/10448630500454361

15. A. G. Soloviev, T. M. Solovieva, A. V.Stadnik, A. K. Islamov, A. I. Kuklin. JINR Communication. 10, 86 (2003).

16. A. G. Soloviev, T. M. Solovjeva, O. I. Ivankov, D. V. Soloviov, A. V. Rogachev, A.I. Kuklin. IOP Conf. Series: Journal of Physics: Conf. Series - YuMO2016. 848(012020), 1 (2017). DOI: 10.1088/1742-6596/848/1/012020

17. M.L. Bernshtejn, L.M. Kaputkina, S.D. Prokoshkin. Tempering of steel. Moscow, MISIS (1997) 336 p. (in Russian) [М.Л. Бернштейн, Л.М.Капуткина, С.Д. Прокошкин. Отпуск стали. Москва, МИСИС (1997) 336 c.]

18. M.A. Smirnov, V.M. Schastlivcev, L.G. Zhuravlev. Basics of heat treatment of steel. Moscow, Nauka \& Technology (2002) 519 p. (in Russian) [М.А. Смирнов, В. М. Счастливцев, Л.Г. Журавлев. Основы термической обработки стали. Москва, Наука и технологии (2002) 519 c.]

19. E.M. Grinberg, A.A. Alekseev, A.V. Budarina, M.S. Salomatnikov. Izvestiya Tula State University. Technical science. 5.2, 251 (2015) (in Russian) [Е.М. Гринберг, А.А. Алексеев, А.В. Бударина, М.С. Саломатников. Известия ТулГУ. Технические науки. 5.2, 251 (2015).]

20. T.A. Lychagina, A.A. Zisman, E.A. Yashina, D. I. Nikolayev. Advanced Engineering Materials. 1700559, 1 (2017). DOI: 10.1002/adem.201700559 